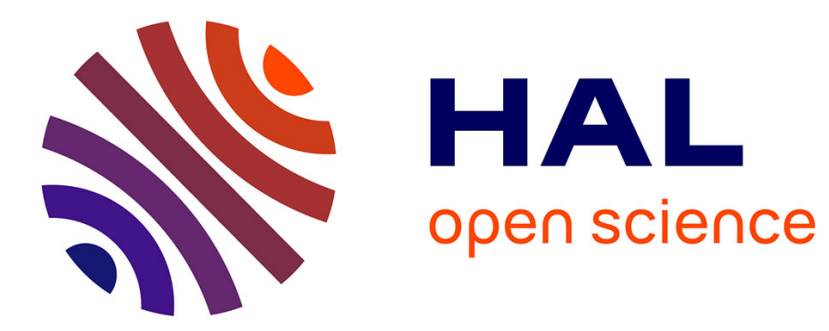

\title{
Simple frustrated systems: chains, strips and squares
}

Bernard Derrida, J. Vannimenus, Y. Pomeau

\section{To cite this version:}

Bernard Derrida, J. Vannimenus, Y. Pomeau. Simple frustrated systems: chains, strips and squares. Journal of Physics C: Solid State Physics, 1978, 11 (23), pp.4749-4765. 10.1088/00223719/11/23/019 . hal-03285942

\section{HAL Id: hal-03285942 \\ https://hal.science/hal-03285942}

Submitted on 21 Jul 2021

HAL is a multi-disciplinary open access archive for the deposit and dissemination of scientific research documents, whether they are published or not. The documents may come from teaching and research institutions in France or abroad, or from public or private research centers.
L'archive ouverte pluridisciplinaire HAL, est destinée au dépôt et à la diffusion de documents scientifiques de niveau recherche, publiés ou non, émanant des établissements d'enseignement et de recherche français ou étrangers, des laboratoires publics ou privés. 


\title{
Simple frustrated systems: Chains, strips and squares
}

\author{
B Derrida†, J Vannimenus \\ + Institut Laue-Langevin, BP 156, 38042 Grenoble Cedex, France \\ ¥ Laboratoire de Physique de l'Ecole Normale Supérieure, 75231 Paris Cedex 05, France \\ \$ Département de Physique Théorique, CEN Saclay, BP 2, 91190 Gif sur Yvette, France
}

Received 8 May 1978

\begin{abstract}
The ground-state properties of several systems showing the Toulouse frustration effect are investigated analytically. First, the energy and entropy of the random-bond Ising chain in a uniform field are obtained for all concentrations of antiferromagnetic bonds, using the transfer-matrix method. The susceptibility has discontinuities for an infinite number of critical values of the field. where the entropy shows spikes in addition to discontinuities. We relate these effects to the physics of frustration. The second system studied consists of frustrated strips, which we argue are the proper one-dimensional limit of spin glasses. Two kinds of strips are considered and the results are compared with recent numerical works on two-dimensional spin glasses and with the exact results for random $(3 \times 3)$ squares.
\end{abstract}

\section{Introduction}

A new fundamental concept, frustration, was recently introduced to interpret the remarkable properties of such disordered systems as spin glasses (Toulouse 1977). As emphasised by Toulouse, it is necessary to study the frustration effect in its own right, at first on simple cases unobscured by all the intricacies of real spin glasses. Like percolation, its older counterpart, the frustration effect raises a large number of questions: What are the numerical values for the critical concentration of competing bonds? How can one define critical exponents? What scaling laws do they obey? How do they depend on spin and lattice dimensionality? Up to now, most work on the problem has relied on numerical methods (Bray et al 1978, Kirkpatrick 1977, Vannimenus and Toulouse 1977). It appears very difficult to find solvable models other than the completely frustrated 'odd model' (Villain 1977).

The natural idea is to look at one-dimensional models, but a chain of spins with randomly ferromagnetic and antiferromagnetic bonds is not frustrated. A simple change of variables makes the problem trivial and this approach looks unprofitable at first sight. The same situation exists in percolation theory for a chain with randomly cut bonds, since one cut suffices to disconnect the chain. To obtain an interesting one-dimensional problem, one has to modify the original problem, for instance by introducing a 'ghost site' (Reynolds et al 1977). In this way one obtains critical exponents in one dimension, which verify the scaling laws for percolation. In the present paper we follow a similar line of thought, and study some models which are essentially one-dimensional and still exhibit non-trivial frustration effects. 
The first model we consider is the random-bond chain of Ising spins in a uniform magnetic field, at zero temperature and for an arbitrary concentration of antiferromagnetic bonds. At a series of field strengths, it becomes favourable to flip well-defined spin clusters and the magnetisation jumps suddenly. In general, there are several equivalent choices for the spins to flip, because it is not possible to satisfy every bond at the same time. This is a typical frustration effect and it gives rise to jumps in the entropy of the ground state. The same qualitative features appear in another related model we have studied, the ferromagnetic chain in a random magnetic field.

Next, we consider two random chains of Ising spins coupled by random bonds, with periodic boundary conditions. One can analyse this system in terms of a strip of frustrated plaquettes, following Toulouse (1977). Indeed, such a strip is the real one-dimensional version of the frustration problem, and we compute its energy and entropy for two different assumptions on the bond distribution. Actual calculations are rather intricate and we have only performed them at zero temperature and without external field.

It is tempting to study several coupled chains and see whether any indication of a singularity in the entropy of the 2D frustrated model shows up for a small system. Unfortunately, our methods lead to prohibitively long calculations, so we turned to the simpler case of periodic frustrated squares. We present the results for the ground-state energy and entropy of the 3 by 3 square, which give some insight into the behaviour of the 2D model. Here again a lot of work is needed to proceed to the 4 by 4 square, and these difficulties seem inherent to disordered frustrated systems.

\section{Ising chains}

\subsection{The random chain in a uniform field}

The model is defined by the Hamiltonian:

$$
\mathscr{H}=-\sum J_{i, i+1} S_{i} S_{i+1}-H \sum S_{i}
$$

where the exchange integral $J_{i, i+1}$ equals $J$ with probability $(1-x)$ and $-J$ with probability $x$, and the bond disorder is quenched. The problem has already been studied by several authors, mostly by integral equations or numerical methods (Matsubara 1974, Landau and Blume 1976, Fernandez 1977), but to our knowledge the analytical expression for the magnetisation at arbitrary $x$ and $H$ is not available. The behaviour of the entropy, which is important to understand frustration effects, has not been investigated.

To get some physical understanding of what happens, let us examine a group of three successive antiferromagnetic bonds in an otherwise ferromagnetic chain. If $H$ is larger than $2 J$, all spins must point up. If it is smaller, either of the spins surrounded by two negative bonds flips in order to minimise the total energy, but it is not favourable to flip both spins, so the degeneracy is 2 . To compute the total entropy of the disordered chain, one must take into account all possible configurations. The direct approach is not practical, however, and one has to use the transfer-matrix method. The difficulty then comes from the fact that one has to consider several non-commuting matrices, and study the product of a large number of randomly chosen matrices. For the present problem, the two transfer matrices are:

$$
M_{1}=\left(\begin{array}{ll}
z^{-1+\alpha} & z^{1+\alpha} \\
z^{1-\alpha} & z^{-1-\alpha}
\end{array}\right) \text { with probability } x
$$




$$
M_{2}=\left(\begin{array}{ll}
z^{1+\alpha} & z^{-1+\alpha} \\
z^{-1-\alpha} & z^{1-\alpha}
\end{array}\right) \text { with probability }(1-x)
$$

where $z=\exp (J / k T)$ and $H=\alpha J$. For very low temperatures, it is possible to follow the evolution of the dominant term (in powers of $z$ ) under successive matrix products; this yields the free energy directly. Since the calculations are lengthy, we defer details of the solution to Appendix 1, and only present the results here. The ground-state energy per spin is given by:

$$
E=-J \frac{r^{2} x^{2}+r x(2-x)+(2 x-1)(x-1)+\alpha(1-x)(2 r x+1)}{(1+r x)(1-x+r x)}
$$

and its entropy per spin is:

$$
\frac{S}{k_{\mathrm{B}}}=\frac{x(1-x)^{2}}{(1-x+r x)^{2}} \sum_{n=1}^{\infty}\left(\frac{r x^{2}}{1-x+r x}\right)^{n-1} \ln n .
$$

In these formulae the integer $r$ is defined by

$$
r<2 / \alpha<r+1 \text {. }
$$

Both magnetisation and entropy are constant in the intervals corresponding to successive values of $r$, and they jump when $H=2 J / r$. At these critical values of the field, the arrangement of spins changes and the degeneracy of the ground state is much larger. The entropy is then:

$$
S / k_{\mathrm{B}}=\frac{1-x}{1+(r-2) x} \sum_{t} R_{0}(t) \ln t .
$$

The summation bears over all points $t$ that can be reached from $t_{0}=2$ by the following non-commuting operations:

$$
\left\{\begin{array}{l}
t \rightarrow T_{1}(t)=1+t \\
t \rightarrow T_{2}(t)=1+1 / t
\end{array}\right.
$$

with

$$
\begin{aligned}
& R_{0}(2)=x^{2}(1-x) /[1+(r-1) x] \\
& R_{0}\left[T_{1}(t)\right]=R_{0}(t) x^{2}(r-1) /[1+(r-2) x] \\
& R_{0}\left[T_{2}(t)\right]=R_{0}(t) x(1-x) /[1+(r-2) x] .
\end{aligned}
$$

For $H=2 J(r=1)$, only $T_{2}$ contributes and formula (5) reduces to a simple series; its value is 0.1428 for $x=0.5$, much larger than the value obtained from equation 4 .

The same kind of generalised series also appears in the strip problem and it seems likely that this is a general feature of problems involving products of random noncommuting matrices. Necessary details on their derivation are given in the Appendix and here we only present some comments on the results displayed in figures 1 to 4 for a few selected values of the parameters:

(i) All properties are continuous with respect to the concentration $x$ of negative bonds, and for $x=0.5$ the magnetisation is in agreement with the low-temperature numerical results (Fornandez 1977), except in the very low-field region which the numerical method could not study in detail. 


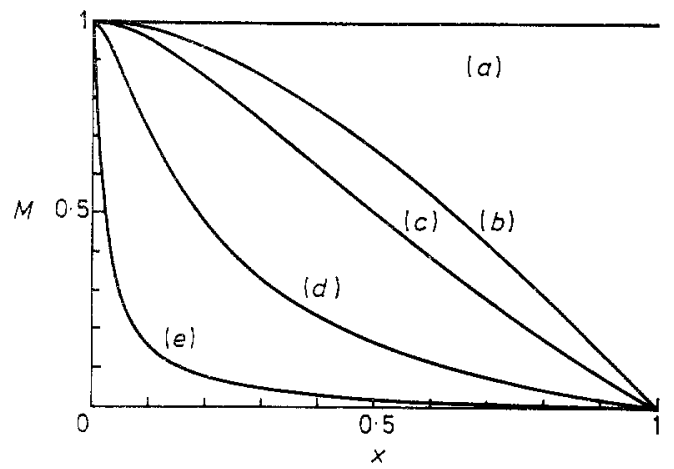

Figure 1. Magnetisation of the random-bond Ising chain in a uniform field $H$ as a function of the concentration $x$ of antiferromagnetic bonds at zero temperature. The different curves correspond to a range of values of $H: 2 / r>H / J>2 /(r+1)$ for $r=0(a), 1(b), 2(c), 10(d)$, $100(e)$.

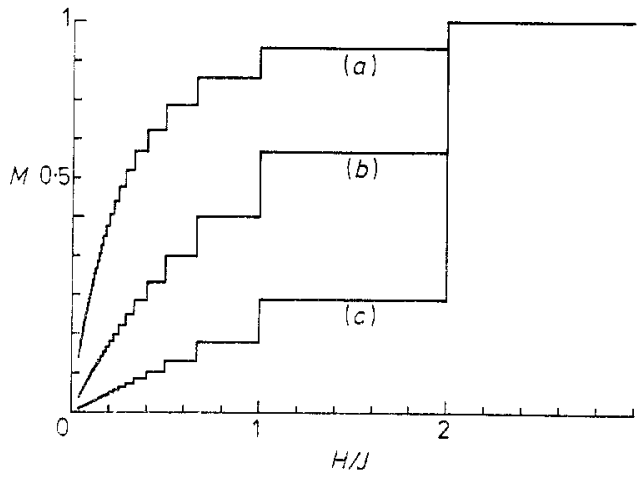

Figure 2. Magnetisation as a function of $H$ for different concentrations $x=0 \cdot 2(a), 0 \cdot 5(b)$, $0.8(c)$. When $H$ tends to zero, the magnetisation of the chain shows an infinite number of discontinuities but the susceptibility has a finite limit (equation 6).

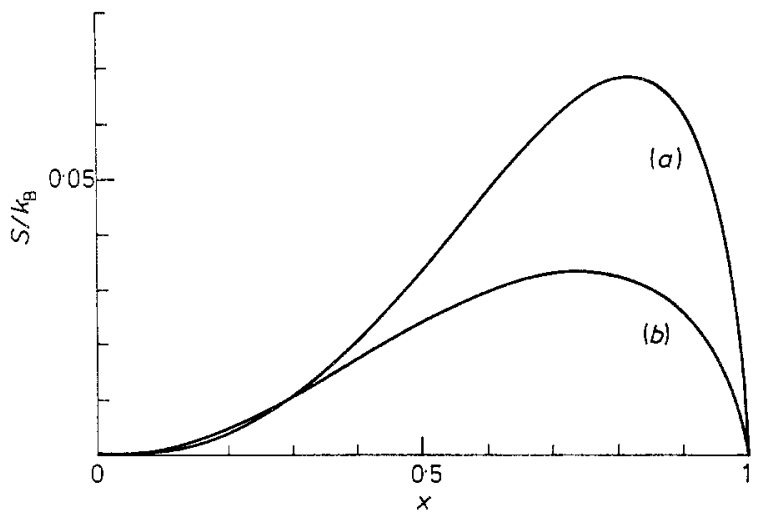

Figure 3. Entropy of the random-bond chain as a function of $x$ for two ranges of values of the field: $2>H / J>1(a) ; 1>H / J>\frac{2}{3}(b)$. The slope $\mathrm{d} S / \mathrm{d} x$ is infinite for $x=1$ (equation 7). Note that at low $x$, the entropy is smaller in a larger field. 


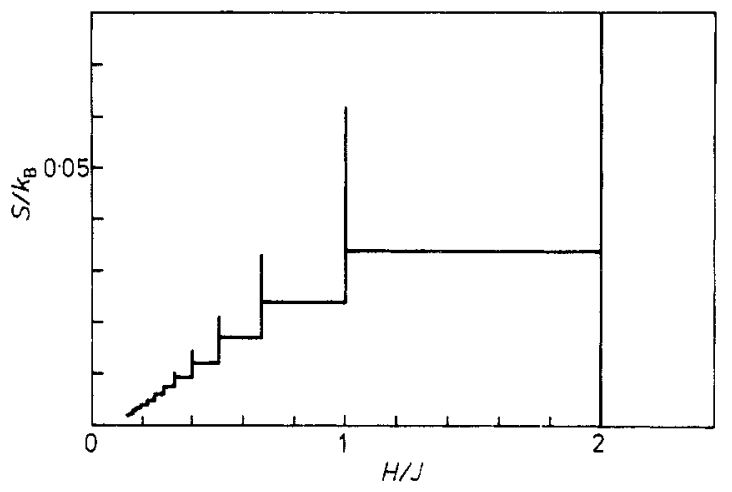

Figure 4. Entropy as a function of $H$ for $x=0.5$. The spikes for the critical values $(2 J / H=r)$ are drawn only up to $r=6$ for clarity. For $r=1$, the numerical value is $S / k_{\mathrm{B}}=0.143$ and lies outside the figure.

(ii) For very small values of the field, the magnetisation at fixed $x$ undergoes an infinite number of discontinuities (figure 2) but the susceptibility has a finite limit (at $T=0)$ :

$$
(H \rightarrow 0) \chi(0)=\lim (M / H)=(1-x) / x .
$$

At finite $T$, the susceptibility is just $(J / k T)$ ih zero field (Bray et al 1978 ), so it is not continuous when $T$ tends to zero. In other words, the limits $T \rightarrow 0$ and $H \rightarrow 0$ do not commute for the random-bond chain. This behaviour is linked to the existence of very large clusters of spins that flip in very small external fields and it may be regarded as a quasi-collective effect.

(iii) The entropy shows a broad maximum for rather large values of $x$, at a given field, and it vanishes with infinite slope when the chain becomes antiferromagnetic. The general term in the series (equation 4) behaves as $x^{n} \ln n$ and one finds for the singular part:

$$
(1-x \ll 1) \frac{S}{k_{\mathrm{B}}} \sim-\frac{(1-x) \ln (1-x)}{r(1+r)} .
$$

The non-analyticity of $S$ is a remarkable feature, which is also linked to large clusters, as is readily seen from the series expansion (equation 4).

(iv) The spikes in the entropy for integer values of $(2 J / H)$ are quite high and correspond to a very large degeneracy (figure 4). The complicated form of their analytical expression (equation 5) shows they are not just due to a simple set of configurations as in the general case. That such effects are physical may be directly seen in the pure antiferromagnet where the calculations are tractable for all $H$ and $T$ : the entropy is continuous in $T$ and at $T=0$, it vanishes except for $H=2 J$ where its value is $\ln [(1+\sqrt{5}) / 2]$. More generally, for non-zero $T$, the discontinuities will be smoothed out and the spikes will turn into sharp maxima, indicating strong changes in local ordering at the critical values of $(H / J)$ though no real transitions subsist in one dimension.

\subsection{The ferromagnetic chain in a random field}

In the absence of an external field, the random-bond chain is equivalent to a ferromagnetic chain, through a simple change of variables. However, the pure chain in a field of fixed 
magnitude and random sign is not in general equivalent to the random chain in a uniform field. The calculations are somewhat more complicated but follow the same lines as in the preceding section and we do not give them here. Keeping the same notation ( $x$ is now the probability of a negative field), one obtains for the energy per spin:

$$
E=-J\left\{1+x\left[\alpha p_{0}+(1+r) \alpha q_{r}-2 q_{r}\right]+(1-x)\left[\alpha q_{0}+(1+r) \alpha p_{r}-2 p_{r}\right]\right\}
$$

and for the entropy:

$$
\frac{S}{k_{\mathrm{B}}}=2 q_{r} x\left(1-x \frac{p_{1}}{p_{0}}\right) \sum_{n=1}^{\infty}\left(x \frac{p_{1}}{p_{0}}\right)^{n-1} \ln n
$$

with

$$
\begin{aligned}
& \beta=(1-2 x) x^{2 r+2} /\left[(1-x)^{2 r+3}+x^{2 r+3}-x^{r+1}(1-x)^{r+1}\right] \\
& p_{i}(x)=q_{i}(1-x)=\beta\left[(1-x)^{1+r} / x^{1+r}-(1-x)^{i} / x^{i}\right] .
\end{aligned}
$$

These formulae are invariant when $x$ is changed in $(1-x)$, as it should be, and they simplify in the symmetric case $\left(x=\frac{1}{2}\right)$, which is entirely equivalent to the corresponding case for the random-bond problem. The qualitative behaviour of the model is very similar to that of the random chain for all values of $x$, with discontinuities in magnetisation and entropy at critical values of $H$. The model may be useful to understand qualitatively the response of an ordered system to a finite random field, and to assess the validity of usual perturbation expansions.

These discontinuities also exist at $T=0$ for $2 \mathrm{D}$ systems and some of them may give rise to transition lines at finite $T$, as is observed for the pure 2D antiferromagnet in a uniform field. So we may hope that the behaviour of chains at zero $T$ gives some insight into the behaviour of disordered $2 \mathrm{D}$ systems at non-zero temperatures.

\section{Frustrated strips}

\subsection{Description of the models}

In the study of coupled random-bond Ising chains, it is convenient to impose periodic boundary conditions; this is equivalent to wrapping the chains on a cylinder. To make the model as simple as possible we choose equal strengths and equal probabilities for intrachain and interchain bonds. Then, the ground states may be analysed in terms of frustration: every time the number of negative bonds around an elementary square ('plaquette') is odd, it is not possible to satisfy all four bonds at the same time and the plaquette is frustrated. For Ising spins, a ground state is determined by a pairing of such plaquettes, with strings of minimal total length (Toulouse 1977).

We have considered two possibilities for the distribution of random bonds (figure 5):

(i) the symmetric strip (ss) for which both interchain couplings in a given column are constrained to be equal. Due to this restriction, the pattern of frustrated plaquettes is symmetric and purely one-dimensional (case A).

(ii) the completely random strip (CRS) for which all couplings are independent random variables. In this case, the pattern has a bit of $2 \mathrm{D}$ character (case $\mathrm{B}$ ) and the comparison with case $\mathrm{A}$ is instructive. 


\subsection{The symmetric strip}

The frustrated plaquettes always appear in blocks on the ss (figure 5). In a ground state, such blocks cannot be linked by frustration strings and they are independent. Our task is then reduced to obtaining the energy, degeneracy and probability of individual blocks. This may be rather easily accomplished by solving recurrence equations on the block size and taking into account the periodic boundary conditions on the spins.

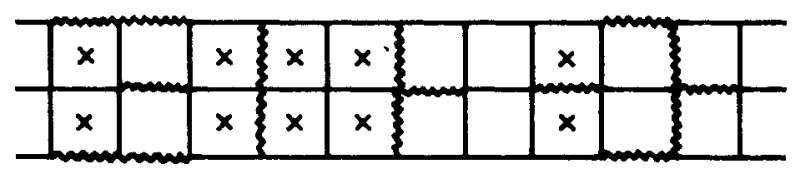

(a)

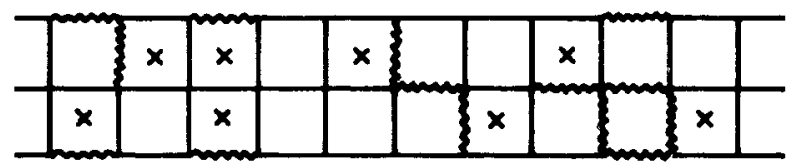

(b)

Figure 5. Schematic diagrams for random strips. The wavy lines represent antiferromagnetic bonds, and the strips are periodic, i.e. the top and bottom rows of spins are identical. When the number of negative bonds around an elementary square ('plaquette') is odd, the four bonds cannot all be satisfied. The plaquette is frustrated and denoted by a cross. In model A (a), both vertical bonds in a given column are equal, so the pattern of frustration is symmetric (symmetric strip model). In model B (b), no restriction is placed upon the bonds (completely random strip model).

The final result for the probability of a block of size $n$ (i.e. containing $2 n$ frustrated plaquettes) reads:

$$
P_{n}=\left(\lambda_{+}-1\right)^{2} \lambda_{+}^{\eta} P_{+}(z)+\left(\lambda_{-}-1\right)^{2} \lambda_{-}^{n} P_{-}(z)
$$

with

$$
\begin{aligned}
& z=1-2 x \\
& \lambda_{ \pm}=\left[1-z^{2} \pm\left(1+2 z^{2}-3 z^{4}\right)^{1 / 2}\right] / 4
\end{aligned}
$$

and

$$
P_{ \pm}=\frac{1}{2} \pm \frac{1+z^{2}-2 z^{4}}{2\left(1+2 z^{2}-3 z^{4}\right)^{1 / 2}}
$$

The ground-state entropy per spin is here:

$$
S / k_{\mathrm{B}}=\Sigma P_{n}(z) \ln W_{n}
$$

where $W_{n}=\left[(1+\sqrt{ } 2)^{n}(2+\sqrt{ } 2)+(1-\sqrt{ } 2)^{n}(2-\sqrt{ } 2)\right] / 4$.

For $x=\frac{1}{2}(z=0)$, its numerical value is 0.199 . As for the energy, it is simply found by noting that one bond is frustrated for every pair of plaquettes. The density $c_{p}$ of frustrated plaquettes equals $\left(1-z^{4}\right) / 2$ and the ground-state energy per spin is:

$$
E / J=-2+c_{\mathrm{p}} / 2=-\left(3+z^{4}\right) / 2 \text {. }
$$


We defer the discussion to the next section, where we compare the results with the CRS case. Other important quantities are the correlation functions, but the usual correlation $\overline{\left(S_{i} S_{i+n}\right)}$ is not simply related to the frustration of the strip. Rather, the quantity of direct interest here is the correlation function defined by:

$$
\left.q(n)=N^{-1} \sum_{i} \overline{\left(S_{i} S_{i+n}\right.}\right)^{2}
$$

where the bar corresponds to an average taken over all ground states for a given distribution of bonds. What $q(n)$ measures is the degree of solidarity of spins $n$ sites apart, irrespective of their orientations: it is equal to 1 if these spins always keep the same relative orientations, it is 0 if they have the same probability of being parallel as antiparallel.

It proves very difficult to obtain $q(n)$ and instead, we focus on another related quantity, the distribution of 'packets' of solidary spins. A 'packet' $\dagger$ is defined as a cluster of spins which always keep the same relative orientation in all possible ground states. Their internal solidarity is complete, but different packets may have some solidarity, and $q(n)$ might have a non-zero limit for large $n$ even though there is no infinite packet. In the present case, spins separated only by non-frustrated plaquettes belong to the same packet, and spins separated by an odd block of frustrated plaquettes have no correlation at all.

For commodity, we define $B_{n}$ as the probability of a sequence of exactly $n$ nonfrustrated pairs of plaquettes, i.e. a packet of $(2 n+2)$ spins (for $n \neq 0$ ). Using similar recurrence methods, we obtain:

$$
B_{n}=\left(\mu_{+}-1\right)^{2} \mu_{+}^{n} q_{+}(z)+\left(\mu_{-}-1\right)^{2} \mu_{-}^{n} q_{-}(z)
$$

where

$$
\begin{aligned}
& \mu_{ \pm}(z)=\left[1+z^{2} \pm\left(1-2 z^{2}+5 z^{4}\right)^{1 / 2}\right] / 4 \\
& q \pm(z)=\frac{1}{2}\left[1 \pm \frac{1-z^{2}+2 z^{4}}{\left(1-2 z^{2}+5 z^{4}\right)^{1 / 2}}\right]
\end{aligned}
$$

Two limits have special interest. The probability that a spin is isolated is

$$
B_{0}=\left[1-2 z^{4}+z^{6}\right] / 4
$$

and when the concentration $x$ is small, the probability of large packets occuring is:

$$
B_{n} \sim 9 x^{2} \exp (-3 n x) \quad(n \gg 1)
$$

The factor $(3 x)$ just comes from the three independent bonds at each step, and these results may be regarded as the analogue for frustration of the cluster size distribution studied in percolation theory (Wolff and Stauffer 1978). Adopting the same notation, we see that $B_{n}$ is of the form:

$$
B_{n} \sim C n^{-\tau} f\left[n^{\sigma}\left(x-x_{c}\right)\right]
$$

with

$$
x_{\mathrm{c}}=0, \quad \tau=2, \quad \sigma=1 .
$$

† The term 'packet' has been proposed by G Toulouse (Toulouse 1978). 


\subsection{The completely random strip}

In the CRS, it is no longer possible to isolate groups of frustrated plaquettes and enumerate all configurations (figure 5). We must then use the transfer-matrix method described in Appendix 2. The calculations are essentially identical to the random-chain problem, but much more tedious because they involve 16 random matrices. The three-chain problem involves 64 random matrices and looks too big a job, considering that no definite answer would emerge, but just a trend in ground-state properties (for the $2 \mathrm{D}$ problem).

The ground-state energy is most simply expressed with the variable $z=1-2 x$ :

$$
\frac{E}{J}=-\frac{1}{4} \cdot \frac{10-3 z^{2}+8 z^{4}+z^{6}}{2-z^{2}+z^{4}} \text {. }
$$

It is higher than the energy of the symmetric strip, because it is no longer possible to pair only adjacent frustrated plaquettes. Comparison with numerical results in $2 \mathrm{D}$ (Kirkpatrick 1977, Vannimenus and Toulouse 1977) shows that the 2D energy lies between this result and the ss energy (equation 12), which always constitutes a lower bound. The ground-state entropy reads:

$$
S / k_{\mathrm{B}}=\frac{1}{2} \Sigma\left[R\left(T_{2}(t)\right)+Q\left(T_{2}(t)\right)\right] \ln t
$$

Here the summation is over all points $t$ that can be reached from $t_{0}=2$ by the following operations:

$$
\begin{aligned}
& t \rightarrow T_{1}(t)=2+t \\
& t \rightarrow T_{2}(t)=2+1 / t .
\end{aligned}
$$

The weights $R$ and $Q$ are rational fractions in $x$ and their explicit expression is given in the Appendix.

For $x=0 \cdot 5$, the numerical value of $S$ is $0 \cdot 156$, to be compared with the $2 \mathrm{D}$ estimates, which lie between 0.08 and $0 \cdot 10$. In figure 6 , we have plotted the derivatives $\mathrm{d} S / \mathrm{d} x$

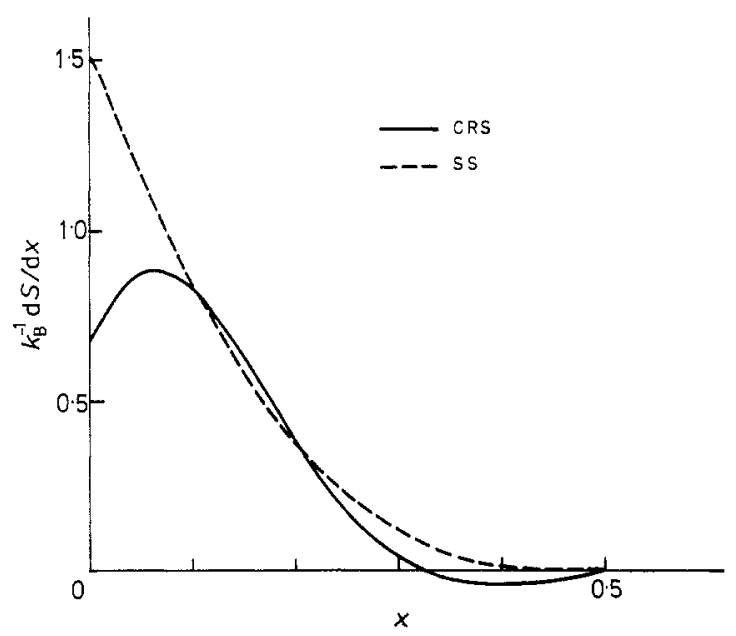

Figure 6. Derivative of the entropy of random strips as a function of $x$ (the curves for $x>0.5$ may be obtained by symmetry). Note that $\mathrm{d} S / \mathrm{d} x$ is monotonic for the symmetric strip (ss), whereas it shows a maximum at $x \sim 0.06$ for the completely random strip (CRS). This effect is related to the incipient two-dimensional character of the latter model. 
rather than the entropies, in order to point out what we believe is a significant trend. In the CRS model, a maximum appears in $\mathrm{d} S / \mathrm{d} x$ for $x \sim 0.06$, and we suggest that for a larger number of chains, this maximum shifts to a larger value of $x$ and sharpens progressively, finally turning into a singularity at $x_{c}$ for the two-dimensional model. The other noteworthy feature is the negative part in $\mathrm{d} S / \mathrm{d} x$ for $x$ above $0 \cdot 32$ : in this region, increasing $x$ decreases the degeneracy of the ground states and the symmetric distribution of bonds $(x=0.5)$ does not correspond to the largest disorder: detailed study shows that the leading term in $S$ near $x=0.5$ is in $z^{2}$, as in the energy. Such terms arise here because the shortest frustrated closed path on the strip contains two bonds and has probability $z^{2}$; this pecularity is due to the periodic boundary conditions and does not exist for systems with four chains and more. We therefore think the paradoxical effect is due to the boundary conditions, although we have no simple physical explanation for it. Incidentally, the same reasoning proves that for $2 \mathrm{D}$ systems these two quantities have a leading term in $z^{4}$ near $x=0 \cdot 5$, which means linearity in the concentration of frustrated plaquettes, as is observed in numerical experiments.

\section{Frustrated squares}

Since our efforts to study a larger number of coupled chains were frustrated, we turned to the simpler problem of finding the ground-state properties of random squares. To take full advantage of the frustration concept, we always consider bonds of equal strength and random sign and we also impose boundary conditions of a slightly different nature. Instead of periodic conditions on the spins, we impose them on the frustrated bonds: the spins may have a periodic or antiperiodic arrangement on the fundamental square. These conditions are much more convenient for our purpose, because it is possible to forget completely the spins and considerably reduce the number of different configurations to be studied; moreover, the effective sample size is larger, and the boundary conditions do not favour ferromagnetic order over disordered states.

Practically, the task consists of classifying all different configurations of frustrated plaquettes, using symmetries and periodicity to reduce their number, then computing their probability, energy and degeneracy. The difficulty increases very quickly with size: there are 13 configurations for the $(3 \times 3)$ square and about 200 for the $(4 \times 4)$ square! We have not been able to go beyond the $(3 \times 3)$ case, which we have solved by two independent methods. The first approach uses a computer and directly counts the number of realisations for all thirteen classes. The second approach consists of following the evolution of the configurations when the signs of bonds are randomly changed, and leads to a set of 13 linear differential equations which may be solved analytically. Once the probabilities are obtained, it is straightforward to compute any quantity of interest. In particular, the energy and entropy per spin are given by:

$$
\begin{aligned}
& E=-(J / 64)\left(93+25 z^{4}+16 z^{6}+7 z^{8}-16 z^{10}+3 z^{12}\right) \\
& S / k_{\mathrm{B}}=((\ln 2) / 96)\left(9-12 z^{4}-8 z^{6}-3 z^{8}+24 z^{10}-10 z^{12}\right) .
\end{aligned}
$$

Such polynomial ex pressions cannot lead to any singularity, but it is instructive to compare them with the strip and $2 \mathrm{D}$ results. The energy is slightly higher than the ss lower bound and lower than the 2D case, and it does not show any remarkable feature. The entropy is more interesting (figure 7), it starts as $6 x^{2} \ln 2$ like the $2 \mathrm{D}$ case and has an inflection point at $x=0.092$. For $x$ greater than $0.30, S$ flattens out and the leading term in $z^{4}$ seems to 
differ little from what is found in $2 \mathrm{D}$. These features confirm the trend observed in strips and are consistent with the suggested existence of a singularity in $d S / d x$ in two dimensions.

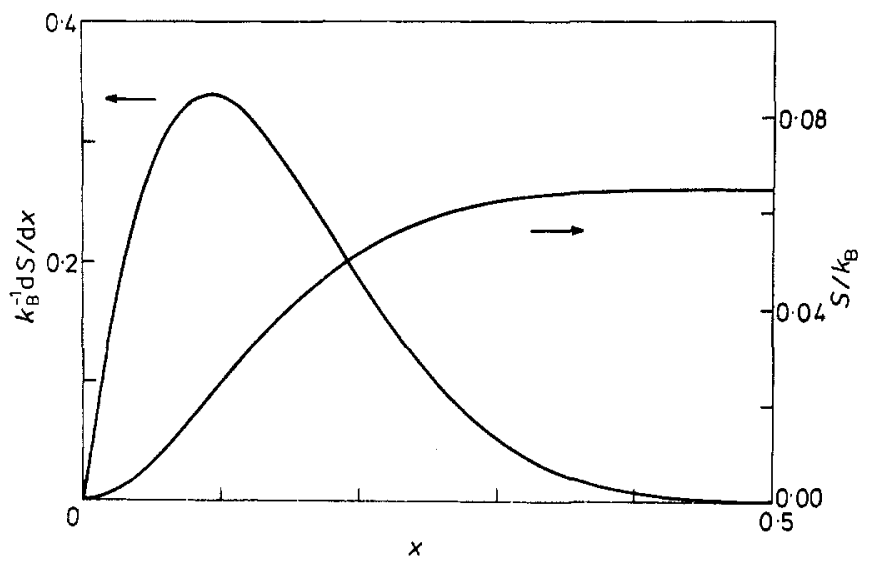

Figure 7. Entropy and its derivative for the $(3 \times 3)$ random square with periodic conditions on the frustrated bonds (see text). The maximum of $\mathrm{d} S / \mathrm{d} x$ is at $x \sim 0.09$.

\section{Conclusion}

We have presented an analytic study of ground-state properties for several simple models with quenched disorder. This work will look somewhat disparate in its aims and methods, but our motivation is to gain a better understanding of one unifying concept, frustration, and we think a few general conclusions emerge. Firstly, these problems are non-trivial in spite of their essentially one-dimensional character and the restriction to ground-state properties. Secondly, the entropy is the most interesting physical quantity in frustrated systems: the hallmark of frustration is to push up together, to equivalent energies, very different states that would be well separated in the ordered system, and that effect is most obvious in the entropy. By contrast, the ground-state energy is affected in a much smoother way by the existence of frustration. At several places, we have pointed out the possibility that some of the observed effects subsist in systems of higher dimensionality at finite temperatures, and that the one-dimensional systems will fit naturally into a general pattern for frustrated systems. Finally, we hope these kinds of non-trivial models may be used as a testing ground for renormalisation-group or decimation methods and will prove richer than the simple disordered Ising chain for this purpose.

\section{Acknowledgments}

We thank J M Maillard and L De Seze for several useful suggestions and discussions.

\section{Appendix 1}

We want to find the free energy of a random-bond Ising chain in a uniform magnetic 
field $H$. For a chain of $N$ spins, the partition function is:

$$
Z=\operatorname{Tr}\left(\mathscr{H}_{N}\right)
$$

where $\mathscr{M}_{N}$ is a $2 \times 2$ matrix:

$$
\mathscr{M}_{N}=\prod_{i=1}^{N} M_{i}
$$

The matrices $M_{i}$ are randomly chosen:

$$
\begin{aligned}
& M_{i}=\left(\begin{array}{ll}
z^{1+\alpha} & z^{-1+\alpha} \\
z^{-1-\alpha} & z^{1-\alpha}
\end{array}\right) \text { with probability } 1-x \text { (positive bond) } \\
& M_{i}=\left(\begin{array}{ll}
z^{-1+\alpha} & z^{1+\alpha} \\
z^{1-\alpha} & z^{-1-\alpha}
\end{array}\right) \text { with probability } x \text { (negative bond) }
\end{aligned}
$$

where $z=\exp \left(J / k_{\mathrm{B}} T\right)$ and $\alpha=V / J$.

To find the free energy per spin, in the thermodynamic limit, one has to calculate:

$$
\left\langle\lim _{N \rightarrow \infty} 1 / N \ln \left(\operatorname{Tr} \mathscr{M}_{N}\right)\right\rangle
$$

where the average is taken over all the possible choices of matrices $M_{i}$. As these matrices have all their elements positive, one can verify that:

$$
\lim _{N \rightarrow \infty}\left\langle 1 / N \ln \left(\operatorname{Tr} \mathscr{M}_{N}\right)\right\rangle=\lim _{N \rightarrow \infty}\left\langle 1 / \ln \left(\text { any element of } \mathscr{M}_{N}\right)\right\rangle .
$$

We perform the calculation only in the low-temperature limit, which means that we keep for each element of $\mathscr{M}_{N}$ the leading term in $z$.

Let

$$
\mathscr{M}_{N} \simeq\left(\begin{array}{cc}
z^{a} \mathrm{e}^{u} & z^{b} \mathrm{e}^{v} \\
\ldots & \ldots
\end{array}\right)
$$

when $z \rightarrow \infty$. Then if

$$
\mathscr{M}_{N+1}=\mathscr{M}_{N} M_{N} \simeq\left(\begin{array}{ll}
z^{A} \mathrm{e}^{U} & z^{B} \mathrm{e}^{V} \\
\ldots & \ldots
\end{array}\right),
$$

one can find $A, B, U, V$ knowing $a, b, u, v$ and $M_{N}$.

$$
\mathrm{Call} \begin{cases}C=A-B & W=U-V \\ c=a-b & w=u-v .\end{cases}
$$

With probability $1-x$, one has:

$$
\begin{array}{lll}
\text { If }-2 \leqslant c<2-2 \alpha: & A=a+1+\alpha & U=u \\
& B=b+1-\alpha & V=v \\
& C=c+2 \alpha & W=w . \\
\text { If } c=2-2 \alpha: & A=a+1+\alpha & U=u \\
& B=b+1-\alpha & V=\ln \left(\mathrm{e}^{u}+\mathrm{e}^{v}\right) \\
& C=c+2 \alpha & W=-\ln \left(1+\mathrm{e}^{-w}\right) . \\
\text { If } 2-2 \alpha<c \leqslant 2: & A=a+1+\alpha & U=u \\
& B=a-1+\alpha & V=u \\
& C=2 & W=0 .
\end{array}
$$


With probability $x$, one has:

$$
\begin{array}{lll}
\text { If }-2 \leqslant c<2-2 \alpha: & A=b+1-\alpha & U=v \\
& B=a+1+\alpha & V=u \\
& C=-c-2 \alpha & W=-w . \\
\text { If } c=2-2 \alpha: & A=b+1-\alpha & U=\ln \left(\mathrm{e}^{u}+\mathrm{e}^{v}\right) \\
& B=a+1+\alpha & V=u \\
\text { If } 2-2 \alpha<c \leqslant 2: & C=-2 & W=\ln \left(1+\mathrm{e}^{-w}\right) . \\
& A=a-1+\alpha & U=u \\
& B=a+1+\alpha & V=u \\
& C=-2 & W=0 .
\end{array}
$$

From these recursion relations which define a Markov chain, one can relate the probability density $\rho_{N}(a, b, u, v)$ for matrix $\mathscr{M}_{N}$ to the density $\rho_{N+1}(A, B, U, V)$ for matrix $\mathscr{M}_{N+1}$. The quantity we want to find is:

$$
\begin{gathered}
\lim _{N \rightarrow \infty}\left\langle 1 / N \operatorname{Tr} \mathscr{M}_{N}\right\rangle \simeq \lim _{N \rightarrow \infty} 1 / N \iiint \int(a \ln z+u) \rho_{N} \mathrm{~d} a \mathrm{~d} b \mathrm{~d} u \mathrm{~d} v \\
=\lim _{N \rightarrow \infty}\left(\frac{\langle a\rangle_{N}}{N} \ln z+\frac{\langle u\rangle_{N}}{N}\right) .
\end{gathered}
$$

This will give the ground-state energy and entropy per spin:

$$
\begin{aligned}
& E=J\left(\lim _{N \rightarrow \infty}\langle a\rangle_{N} / N\right)=J \lim _{N \rightarrow \infty}\left(\langle a\rangle_{N+1}-\langle a\rangle_{N}\right) \\
& S / k_{\mathrm{B}}=\lim _{N \rightarrow \infty}\langle u\rangle_{N} / N=\lim _{N \rightarrow \infty}\left(\langle u\rangle_{N+1}-\langle u\rangle_{N}\right) .
\end{aligned}
$$

One can see easily that to calculate $\langle a\rangle_{N+1}-\langle a\rangle_{N}$ or $\langle u\rangle_{N+1}-\langle u\rangle_{N}$, one does not need the complete probability $\rho_{N}(a, b, u, v)$ but it is enough to know a reduced probability $\sigma_{N}(c, w)$ defined by:

$$
\sigma_{N}(c, w)=\iiint \int \rho_{N}(a, b, u, v) \delta(a-b-c) \delta(u-v-w) \mathrm{d} a \mathrm{~d} b \mathrm{~d} u \mathrm{~d} v
$$

When $N \rightarrow \infty$, even though $\rho_{N}$ spreads out, the density $\sigma_{N}$ tends to a limit density $\sigma$, from which the energy and the entropy (A2) may be deduced.

\section{Ground-state energy}

Let us suppose that

$$
\frac{2 J}{r+1}<H<\frac{2 J}{r}, \quad \text { or } \quad \frac{2}{r+1}<\alpha<\frac{2}{r} .
$$

Then the only possible values for $c$ are:

$$
c= \pm(2-2 i \alpha) \text { with } 0 \leqslant i \leqslant r .
$$

Let $p_{i}$ be the probability that $c=-2+2 i \alpha, q_{i}$ be the probability that $c=2-2 i \alpha$.

To find $\sigma$ we must look for a probability distribution invariant under the recursion 
relations. This gives equations for the set of $\left(p_{i}, q_{i}\right)$ :

$1 \leqslant i \leqslant r-1 \quad p_{i}=(1-x) p_{i-1}+x q_{i+1}$

$$
q_{i}=(1-x) q_{i+1}+x p_{i-1}
$$

and

$$
\begin{aligned}
& p_{r}=(1-x) p_{r-1} \\
& q_{r}=x p_{r-1} \\
& p_{0}=x\left(p_{r}+q_{0}+q_{1}\right) \\
& q_{0}=(1-x)\left(p_{r}+q_{0}+q_{1}\right) .
\end{aligned}
$$

One can solve this system:

$$
\begin{array}{ll}
p_{i}=\beta(i+1-r-1 / x) & 0 \leqslant i \leqslant r \\
q_{i}=\beta(i-r-1) & 1 \leqslant i \leqslant r \\
q_{0}=\beta(1-r-1 / x)(1-x) / x &
\end{array}
$$

with $\beta=x^{2} /[(1+r x)(1+r x-x)]$ to ensure the normalisation: $\Sigma_{i}\left(p_{i}+q_{i}\right)=1$.

Using these probabilities and the recursion relations, we can now obtain the ground state energy:

$$
\begin{aligned}
\frac{E}{J}=\lim _{N \rightarrow \infty}\left(\langle a\rangle_{N+1}-\langle a\rangle_{N}\right) & \\
& =(1-x)(1+\alpha)+x \sum_{i=0}^{r-1} p_{i}(2-2 i \alpha+1-\alpha)+x p_{r}(\alpha-1) \\
& +x \sum_{i=1}^{r} q_{i}(-2+2 i \alpha+1-\alpha)+x q_{0}(\alpha-1) \\
& =\frac{r^{2} x^{2}+r x(2-x)+(2 x-1)(x-1)+\alpha[2 r x(1-x)+1-x]}{(1+r x)(1+r x-x)} .
\end{aligned}
$$

One can verify that $\langle b\rangle_{N+1}-\langle b\rangle_{N}$ gives the same result and this agrees with (A1).

\section{Ground-state entropy}

To find the $T=0$ entropy one has to distinguish two cases:

(i) $H \neq 2 J / r$ with $r$ an integer

(ii) $H=2 J / r$.

(i) $2 J /(r+1)<H<2 J / r$. To describe the limit density $\sigma(c, w)$, one notes that the only possible values for $(c, w)$ are:

$$
\left\{\begin{array} { l } 
{ c = - 2 + 2 i \alpha } \\
{ w = \operatorname { l n } n }
\end{array} \quad \text { or } \quad \left\{\begin{array}{l}
c=2-2 i \alpha \\
w=-\ln n
\end{array}\right.\right.
$$

with

$$
0 \leqslant i \leqslant r \text { and } n \text { an integer } \geqslant 1 \text {, }
$$

Let $R_{i}(n)$ be the probability that $c=-2+2 i \alpha, w=\ln n$, and $S_{i}(n)$ be the probability 
that $c=2-2 i \alpha, w=-\ln n$. One has the relations:

$$
\begin{aligned}
& 1 \leqslant i \leqslant r-1\left\{\begin{array}{l}
R_{i}(n)=(1-x) R_{i-1}(n)+x S_{i+1}(n) \\
S_{i}(n)=(1-x) S_{i+1}(n)+x R_{i-1}(n)
\end{array}\right. \\
& R_{r}(n)=(1-x) R_{r-1}(n) \\
& S_{r}(n)=x \quad R_{r-1}(n) \\
& S_{0}(1)=(1-x)\left(p_{r}+q_{0}\right) \\
& R_{0}(1)=x\left(p_{r}+q_{0}\right) \\
& n \geqslant 2 \begin{cases}S_{0}(n)=(1-x) S_{1}(n-1) \\
R_{0}(n)=\quad x \quad & S_{1}(n-1) .\end{cases}
\end{aligned}
$$

The solution of this system is:

$$
\left.\begin{array}{l}
R_{i}(n)=p_{i}(1-\mu) \mu^{n-1} \\
S_{i}(n)=q_{i}(1-\mu) \mu^{n-1}
\end{array}\right\} \text { for } 0 \leqslant i \leqslant r
$$

with $\mu=r x^{2} /(1+r x-x)$.

It is now possible to obtain the entropy:

$$
\begin{aligned}
\frac{S}{k_{\mathrm{B}}}=\lim \left(\langle u\rangle_{N+1}\right. & \left.-\langle u\rangle_{N}\right) \\
& =x \sum_{n=1}^{\infty}\left(\sum_{i=0}^{r-1} R_{i}(n)(-\ln n)+\sum_{i=2}^{r} S_{i}(n) \ln (n)+S_{1}(n) \ln (n+1)\right) \\
& =\frac{x(1-x)^{2}}{[1+(r-1) x]^{2}} \sum_{n=1}^{\infty} \mu^{n-1} \ln n .
\end{aligned}
$$

(ii) $H=2 J / r$. Now the possible values for $c$ and $w$ are:

$$
\begin{array}{ll}
c=-2+2 i \alpha & (0 \leqslant i \leqslant r) \\
w= \pm \ln z &
\end{array}
$$

with probability $R_{i}(z)$ for the $(+)$ case and $S_{i}(z)$ for the $(-)$ case. Here $z$ is a point reached from 1 by the successive action of the functions $T_{1}(z)=1+z$ and $T_{2}(z)=1+1 / z$. The possible values for $z$ are $\left(1,2,3, \frac{3}{2}, 4, \frac{4}{3}, \frac{5}{2} \ldots\right)$. The calculation gives for the probabilities:

$$
\begin{array}{ll}
R_{i}(z)=R_{0}(z)[1+(r-2-i) x] /(1+r x-2 x) & 0 \leqslant i \leqslant r-1 \\
S_{i}(z)=R_{0}(z) i x /(1+r x-2 x) & \\
R_{r}(z)=0 & \\
S_{r}(z)=R_{0}(z)(1-x) / x &
\end{array}
$$

with

$$
\begin{aligned}
& R_{0}(2)=x^{2}(1-x) /(1+r x-x) \\
& R_{0}\left(T_{1}(z)\right)=R_{0}(1+z)=\frac{x^{2}(r-1)}{1+(r-2) x} R_{0}(z) \\
& R_{0}\left(T_{2}(z)\right)=R_{0}\left(1+\frac{1}{z}\right)=\frac{x(1-x)}{1+(r-2) x} R_{0}(z)
\end{aligned}
$$


One can then find $S$, the entropy per spin:

$$
\begin{aligned}
\frac{S}{k_{\mathrm{B}}}=\lim \left(\langle u\rangle_{N+1}\right. & \left.-\langle u\rangle_{N}\right) \\
& =x \sum_{z}\left(\sum_{i=0}^{r-2}\left(S_{i}(z)-R_{i}(z)\right) \ln z+S_{r-1}(z) \ln \left(T_{1}(z)\right)+R_{r-1}(z) \ln \left(T_{2}(z)\right)\right) \\
& =\frac{1-x}{1+(r-2) x} \sum_{z} R_{0}(z) \ln z .
\end{aligned}
$$

In these two cases, one can find the same result by calculating $\langle v\rangle_{N+1}-\langle v\rangle_{N}$ and this agrees with (A1).

\section{Appendix 2}

We give here only the result for the CRs. The calculation can be done as in Appendix 1 and the entropy $S$ is:

$$
S=\frac{1}{2} \sum_{t}\left[R\left(T_{2}(t)\right)+Q\left(T_{2}(t)\right)\right] \ln t
$$

where the sum is done on all the points $t$ reached from 2 by the functions

$$
\begin{aligned}
& T_{1}(t)=2+t \\
& T_{2}(t)=2+1 / t .
\end{aligned}
$$

$R(t)$ and $Q(t)$ are rational fractions in $x:$ if

$$
t=T_{1}^{\alpha} T_{2}^{\beta} T_{1}^{\gamma} T_{2}^{\delta}
$$

then

$$
\left(\begin{array}{l}
R(t) \\
Q(t)
\end{array}\right)=\left(\begin{array}{ll}
B & E \\
E & B
\end{array}\right)^{\alpha}\left(\begin{array}{ll}
F & A \\
D & C
\end{array}\right)^{\beta}\left(\begin{array}{ll}
B & E \\
E & B
\end{array}\right)^{\gamma}\left(\begin{array}{ll}
F & A \\
D & C
\end{array}\right)^{\delta} \ldots\left(\begin{array}{l}
R(2) \\
Q(2)
\end{array}\right)
$$

where

$$
\begin{aligned}
& A=(1-x)^{2}\left(1-2 x+2 x^{2}\right) \\
& B=2 x(1-x)\left(1-2 x+2 x^{2}\right) \\
& C+2 x(1-x)^{3} \\
& D=x^{2}\left(1-2 x+2 x^{2}\right) \\
& E=4 x^{2}(1-x)^{2} \\
& F=2 x^{3}(1-x)
\end{aligned}
$$

and

$$
\begin{aligned}
& R(2)=\frac{A^{2}+A E-F^{2}-F B+F}{1-B+E} \\
& Q(2)=\frac{D^{2}+D E+C-C^{2}-C B}{1-B+E} .
\end{aligned}
$$

For example the first terms in the $x$ expansion of this entropy are:

$S=x \ln 2+x^{2}\left(\frac{5}{2} \ln 5-\ln 2\right)+x^{3}(-42 \ln 2-30 \ln 5+54 \ln 3)+\mathrm{O}\left(x^{4}\right)$. 
Note added in proof. After this work was accepted for publication, we received a related preprint by M Puma and J F Fernandez entitled 'Entropy of a random bond Ising chain' and to be published in Physical Review. The numerical results obtained by these authors (for the case $J_{i j}= \pm J, x=0.5$ at $T \rightarrow 0$ ) agree with our analytical results for the random chain $(\$ 2.1)$. In particular, they observe the sharp maxima in the entropy shown in figure 4 of the present work.

\section{References}

Bray A J. Moore M A and Reed P 1978 J. Phys. C: Solid St. Phys. 111187

Fernandez J F 1977 Phys. Rev. B 165125

Kirkpatrick S 1977 Phys. Rev. B 164630

Landau D P and Blume M 1976 Phys. Rev. B 13287

Matsubara F 1974 Prog. Theor. Phys. 511694

Reynolds P J, Stanley H E and Klein W 1977 J. Phys. A: Math. Gen. 10 L203

Toulouse G 1977 Comm. Phys. 2115

- 1978 Communications: at Les Houches Meeting, March 1978

Vannimenus J and Toulouse G 1977 J. Phys. C: Solid St. Phys. 10 L537

Villain J 1977 J. Phys. C: Solid St. Phys. 101717

Wolff W F and Stauffer D 1978 Z. Phys. B 2967 